\title{
Statistics of amplified light in periodically correlated layered systems with randomness
}

\author{
King-Chuen Chiu and Zhao-Qing Zhang \\ Department of Physics, Hong Kong University of Science and Technology, Clear Water Bay, \\ Hong Kong, People's Republic of China \\ Received 20 January 1997
}

\begin{abstract}
We study the statistics of reflection and transmission coefficients of light in randomly layered amplifying media that are periodic on average. We are interested in one-dimensional universal scaling behaviours in such systems. Our study shows that while a homogeneous medium boundary condition is capable of reproducing universal scaling at low frequencies, a periodic medium boundary condition is necessary for high frequencies. Although the statistics depends on the boundary condition, the saturation length, where the reflection coefficient reaches a stationary distribution, and the localization length do not. Implications of these results are discussed.
\end{abstract}

\section{Introduction}

Wave propagation in random media has been the subject of intensive study during the past decade due to various intensity fluctuation and correlation phenomena arising from the interference between multiply scattered waves [1]. Recently, the interest in this subject has focused on light propagation in amplifying random media because many interesting phenomena arise from the interplay between disorder and amplification [2]. In the presence of gain, the amplitude of a multiply scattered wave of path length $S$ is amplified by a factor $\exp \left(S / l_{\mathrm{g}}\right)$, where $l_{\mathrm{g}}$ is the gain length. Due to this strong path-length dependence, the fluctuation and correlation phenomena found in passive random media are expected to be altered. For instance, the coherent backscattering effect, which arises from the constructive interference between a multiply scattered wave and its time-reversal counterpart, will be amplified more for waves with longer path length. As a result, the coherent backscattering cone becomes sharper in a gain medium [3-6]. Coherent backscattering has its strongest effects in one dimension (1D). It causes all states to be localized. In a homogeneously random 1D system, it has been shown that the enhancement of the coherent backscattering effect is to reduce the localization length [7]. This reduction is also expected in higher dimensions [8]. The probability distribution of the reflection coefficient has been studied analytically in 1D by solving the Riccati equation using a Gaussian white-noise model [9]. This distribution function is found to depend only on two parameters, $L / \xi_{0}$ and $l_{\mathrm{g}} / \xi_{0}$, where $L$ is the sample thickness and $\xi_{0}$ is the localization length in the absence of gain. The scaling is later shown to hold even for the strong scattering and short wavelength limit[7] and for obliquely incident waves as well independent of polarization [10]. Thus, this two-parameter scaling is very robust in 1D and describes a universal scaling behaviour for 1D systems. Moreover, it has been found in [7] that the saturation length, $L_{\mathrm{s}}$, where the distribution 
function reaches a stationary state, follows a scaling relation $L_{\mathrm{s}} \cong\left(\xi_{0} l_{\mathrm{g}}\right)^{1 / 2}$. Recently, this relation has been utilized to provide a method for measuring the localization length by using the resonant tunnelling spectrum [11]. The distribution of the reflection coefficient for multi-channel systems has also been studied in the weak scattering limit [12].

However, most of the studies in 1D have been limited to homogeneously random systems, where the medium parameter, such as the dielectric constant, is assumed to distribute continuously in the medium [7, 9, 10, 12]. Recently, materials made of dielectric composites have been the focus of much study due to the possibility of forming photonic band-gap structures [13]. Most of the systems in this class possess only two medium parameters. In $1 \mathrm{D}$, the localization length behaviour of such random systems that are periodic on average has been studied both theoretically[14] and experimentally $[11,15]$. In the presence of gain, the statistical distribution of the reflection $(R)$ and transmission $(T)$ coefficients and the localization length behaviour of such systems have not been investigated. It is interesting to know if such systems obey the 1D universal scaling found in homogeneously random systems. Since both $R$ and $T$ depend sensitively on the boundary condition, it is of particular importance to understand how the boundary condition affects $R$ and $T$. In the long-wavelength limit, the effective medium theory applies. We would expect that the homogeneous medium boundary condition with an effective dielectic constant will bring the systems to the same scaling behaviours of the homogeneously random systems. However, for high frequencies, particularly those near the band edge where the waves probe the two-component nature of the system, the effective medium theory breaks down and it is not obvious as to what kind of boundary condition is capable of reproducing the 1D universal scaling behaviours. Moreover, since the saturation length, $L_{\mathrm{s}} \cong\left(\xi_{0} l_{\mathrm{g}}\right)^{1 / 2}$, plays an important role in the newly proposed method of measuring the localization length, the question as to whether this relation depends on the boundary condition is crucial to the applicability of this method to real systems [11].

In this work, we address the above two issues. Our numerical simulations show that only a matched boundary condition (MBC) with a periodic embedding medium is capable of reproducing the $1 \mathrm{D}$ universal scaling behaviours at high frequencies. In this case, instead of using plane waves, Bloch waves should be used as the incoming and outgoing waves in measuring the $R$ and $T$. If the embedding medium is homogeneous, we have a mis-matched boundary condition (MMBC). The MMBC gives rise to non-universal behaviours. We have also found that the saturation length obeys $L_{\mathrm{s}} \cong\left(\xi_{0} l_{\mathrm{g}}\right)^{1 / 2}$, independent of the boundary condition. This explains why the method of measuring the localization length proposed earlier can apply to the experimental situations [11].

\section{Model system and mismatched boundary condition}

In a non-magnetic layered medium, the time-independent Maxwell equations for the normally incident light can be written [7] as

$$
\frac{\partial^{2} E(z)}{\partial z^{2}}+\frac{\omega^{2}}{c^{2}} \epsilon(z) E(z)=0
$$

where $\omega, c$ and $E(z)$ are the frequency, the speed of light in a vacuum and the electric field, respectively. We consider a periodic medium consisting of two types of layers with dielectric constants $\epsilon_{A}$ and $\epsilon_{B}$ and layer thicknesses $a$ and $b$. To introduce randomness, instead of making thickness random, without losing generality, we have chosen $\epsilon_{A}$ to be random [14]. Thus, for a disordered sample of $2 L+1$ layers, the thickness $a_{n}$ and the 
dielectric constant $\epsilon_{n}$ of the $n$th layer can be written as

$$
\begin{aligned}
& a_{2 j}=a \\
& a_{2 j+1}=b \\
& \epsilon_{2 j}=\epsilon_{A}\left(1+\sigma \eta_{2 j}\right)-\mathrm{i} \epsilon^{\prime \prime} \\
& \epsilon_{2 j+1}=\epsilon_{B}-\mathrm{i} \epsilon^{\prime \prime}
\end{aligned}
$$

where $j=0,1, \ldots, L . \sigma$ describes the strength of randomness and $\eta_{2 j}$ is a random number between $(-1,1)$. Here, we have assigned the first and last layers to be $B$-type layers. A different arrangement of layer randomness would not alter the results of the calculations. The negative imaginary part of the dielectric constant, i.e. $\epsilon^{\prime \prime}>0$, denotes the coherent amplification of the field, whereas $\epsilon^{\prime \prime}<0$ denotes the attenuation of the field.

In order to study the statistics of the reflection and transmission coefficients we embed the amplifying disordered sample in a passive non-random medium on both sides. If the embedding medium is homogeneous with a dielectric constant $\epsilon_{0}, R$ and $T$ can be calculated by using the transfer-matrix method as described in [7]. The solution of (1) in the $n$th layer can be written as $E(x)=A_{n} \exp \left[\mathrm{i} k_{n}\left(z-z_{n}\right)\right]+B_{n} \exp \left[-\mathrm{i} k_{n}\left(z-z_{n}\right)\right]$, where $k_{n}=(\omega / c) \sqrt{\epsilon_{n}}$ and $z_{n}$ denotes the position of the interface between the $(n-1)$ th and the $n$th layers. For the embedding medium, we have $k_{0}=k_{2 L+1}=(\omega / c) \sqrt{\epsilon_{0}}$. The transfer matrix $\mathcal{T}$, which relates the amplitudes $A_{2 L+1}$ and $B_{2 L+1}$ at the output surface to that at the input surface $A_{0}$ and $B_{0}$, has the form

$$
\left(\begin{array}{l}
A_{2 L+2} \\
B_{2 L+2}
\end{array}\right)=\left(\begin{array}{l}
\mathcal{T}_{11} \mathcal{T}_{12} \\
\mathcal{T}_{21} \mathcal{T}_{22}
\end{array}\right)\left(\begin{array}{l}
A_{0} \\
B_{0}
\end{array}\right)
$$

where $\mathcal{T}=\prod_{i=0}^{2 L+1} \tau^{(i)}$, with

$$
\begin{aligned}
\tau_{11}^{(n)} & =\frac{1}{2}\left(1+\frac{k_{n}}{k_{n+1}}\right) \mathrm{e}^{\mathrm{i} k_{n} a_{n}} \\
\tau_{12}^{(n)} & =\frac{1}{2}\left(1-\frac{k_{n}}{k_{n+1}}\right) \mathrm{e}^{-\mathrm{i} k_{n} a_{n}} \\
\tau_{21}^{(n)} & =\frac{1}{2}\left(1-\frac{k_{n}}{k_{n+1}}\right) \mathrm{e}^{\mathrm{i} k_{n} a_{n}} \\
\tau_{22}^{(n)} & =\frac{1}{2}\left(1+\frac{k_{n}}{k_{n+1}}\right) \mathrm{e}^{-\mathrm{i} k_{n} a_{n}} .
\end{aligned}
$$

By setting $A_{0}=1, B_{0}=r, A_{2 L+2}=t$ and $B_{2 L+2}=0$, we have

$$
\begin{aligned}
& t=\frac{\left(\mathcal{T}_{11} \mathcal{T}_{22}-\mathcal{T}_{12} \mathcal{T}_{21}\right)}{\mathcal{T}_{22}} \\
& r=-\frac{\mathcal{T}_{21}}{\mathcal{T}_{22}} .
\end{aligned}
$$

In our numerical simulations, we have set the speed of light $c$ and layer thicknesses $a$ and $b$ to unity. We have chosen $\epsilon_{B}=1, \epsilon_{A}=2,5$ and 9 . The location of the first band gap in each case is given in table 1. Here we will present the results for the case of small randomness, i.e. $\sigma=0.1$. The effects of randomness on the scaling behaviours will be discussed in section 6. If these systems obey the 1D universal scaling behaviours, the probability distributions of $R\left(=r r^{*}\right)$ and $T\left(=t t^{*}\right)$ should depend on only two parameters, i.e $\Lambda \equiv(2 L+1) / \xi_{0}$ and $q \equiv \xi_{0} / l_{\mathrm{g}}$, and have the same functional forms as 
Table 1. The set of parameters used for the case of a matched boundary condition (MBC).

\begin{tabular}{lllrl}
\hline$\epsilon_{A} / \epsilon_{B}$ & First bandgap & $\omega$ & \multicolumn{1}{l}{$\xi_{0}$} & $\epsilon^{\prime \prime}$ \\
\hline $9 / 1$ & $0.566-0.939$ & 0.35 & 2400 & $2.6806 \times 10^{-3}$ \\
$9 / 1$ & $0.566-0.939$ & 0.4 & 1700 & $3.28794 \times 10^{-3}$ \\
$9 / 1$ & $0.566-0.939$ & 0.5 & 610 & $6.6372 \times 10^{-3}$ \\
$5 / 1$ & $0.761-1.158$ & 0.4 & 3600 & $1.2096 \times 10^{-3}$ \\
$5 / 1$ & $0.761-1.158$ & 0.6 & 1280 & $2.202524 \times 10^{-3}$ \\
$5 / 1$ & $0.761-1.158$ & 1.3 & 690 & $1.221352 \times 10^{-3}$ \\
$2 / 1$ & $1.168-1.437$ & 0.7 & 4050 & $4.3232 \times 10^{-4}$ \\
$2 / 1$ & $1.168-1.437$ & 0.75 & 3600 & $4.535 \times 10^{-4}$ \\
$2 / 1$ & $1.168-1.437$ & 2.4 & 1080 & $4.306 \times 10^{-4}$ \\
\hline
\end{tabular}

those of homogeneously random systems [7,9]. At large $\Lambda$, the distribution of $R, P_{\Lambda}$, should approach the analytical result [9]

$$
P_{\infty}\left(\frac{R-1}{2 q}\right)=\left(\frac{2 q}{R-1}\right)^{2} \exp \left(\frac{-2 q}{R-1}\right) .
$$

Although this analytic result was obtained in the long-wavelength limit, it has been shown numerically in the case of homogeneously random systems that this limiting distribution function is valid even when the wavelength becomes comparable to the averaged layer thickness $d$ as long as the disorder is not too strong so that the localization length in the absence of gain is longer than $100 d$ [7]. It should also be noted that equation (10) does not apply to the case of zero amplification. In this case the distribution function is simply a delta function at $R=1$. Equation (9) becomes valid as soon as any small amount of amplification is introduced $[9,10]$.

For each set of parameters $\epsilon_{A} / \epsilon_{B}, \omega$ listed in table 1 , and $\sigma=0.1$, the values of $L$ and $\epsilon^{\prime \prime}$ are set by the values of $\Lambda$ and $q$, respectively. In order to make comparison with the results obtained in [7] for the case of homogeneously random systems, the values of $q$ have been chosen in the range 0.1 to 10 . The localization length in the absence of gain is calculated from $1 / \xi_{0}=\langle-\ln (T)\rangle / 2(2 L+1)$ in the large- $L$ limit, whereas the relation between $\epsilon^{\prime \prime}$ and $l_{\mathrm{g}}$ can be obtained from the exponential growth of $T$ when $\sigma=0$. In each case, the distributions of $R$ and $T$ are obtained from 10000 configurations.

Our simulation results show that the scaling holds only in the low-frequency, or longwavelength $(\lambda=2 \pi / \omega \gg 2)$ limit, and small-contrast $\left(\epsilon_{A}=2\right)$ limit when an effective medium dielectric constant $\epsilon_{0}=\left(\epsilon_{A}+\epsilon_{B}\right) / 2$ is used in the embedding medium. As the frequency is closer to the band gap, the waves probe the two-component nature of the medium and the scaling breaks down. For instance, at those frequencies listed in table 1, the function $P_{\Lambda}$ converges to forms other than (9) at large $\Lambda$. These frequencies locate near both sides of the first band gap. The corresponding values of $\xi_{0}$ used for rescaling are also given in table 1 .

\section{Matched boundary condition}

In order to bring the system to the univeral scaling behaviour for all frequencies, the embedding medium should also possess the two-component nature. Therefore, we embed the sample in a pure periodic passive medium. In this matched boundary condition (MBC) case, the Bloch wavefunctions should be used to define the reflection $(\bar{r})$ and transmission $(\bar{t})$ amplitudes. Let $\psi^{+}$and $\psi^{-}$be the right-going and left-going Bloch waves, respectively. 
We can write

$$
\psi^{ \pm}(z)=\mathrm{e}^{ \pm \mathrm{i} K z} u(z)= \begin{cases}A^{ \pm} \mathrm{e}^{\mathrm{i} k_{A} z}+B^{ \pm} \mathrm{e}^{-\mathrm{i} k_{A} z} & \text { if } z \in A \\ C^{ \pm} \mathrm{e}^{\mathrm{i} k_{B} z}+D^{ \pm} \mathrm{e}^{-\mathrm{i} k_{B} z} & \text { if } z \in B\end{cases}
$$

where $K$ is the Bloch wavevector, $k_{A}=\omega \sqrt{\epsilon_{A}} / c, k_{B}=\omega \sqrt{\epsilon_{B}} / c$, and $u(z)$ is a periodic function of period $d(=a+b)$. The dispersion relation and the amplitudes $A^{ \pm}, B^{ \pm}, C^{ \pm}$ and $D^{ \pm}$are determined by the secular equation [16]

$$
\cos (K d)=\cos \left(k_{A} a\right) \cos \left(k_{B} b\right)-\frac{k_{A}^{2}+k_{B}^{2}}{2 k_{A} k_{B}} \sin \left(k_{A} a\right) \sin \left(k_{B} b\right)
$$

and

$$
\left(\begin{array}{cccc}
1 & 1 & -\mathrm{e}^{\mathrm{i} d\left(k_{B} \mp K\right)} & -\mathrm{e}^{-\mathrm{i} d\left(k_{B} \pm K\right)} \\
k_{A} & -k_{A} & -k_{B} \mathrm{e}^{\mathrm{i} d\left(k_{B} \mp K\right)} & k_{B} \mathrm{e}^{-\mathrm{i} d\left(k_{B} \pm K\right)} \\
\mathrm{e}^{\mathrm{i} k_{A} a} & \mathrm{e}^{-\mathrm{i} k_{A} a} & -\mathrm{e}^{\mathrm{i} k_{B} a} & -\mathrm{e}^{-\mathrm{i} k_{B} a} \\
k_{A} \mathrm{e}^{\mathrm{i} k_{A} a} & -k_{A} \mathrm{e}^{-\mathrm{i} k_{A} a} & -k_{B} \mathrm{e}^{\mathrm{i} k_{B} a} & k_{B} \mathrm{e}^{-\mathrm{i} k_{B} a}
\end{array}\right)\left(\begin{array}{c}
A^{ \pm} \\
B^{ \pm} \\
C^{ \pm} \\
D^{ \pm}
\end{array}\right)=\left(\begin{array}{l}
0 \\
0 \\
0 \\
0
\end{array}\right) .
$$

Since the layers adjacent to the disordered sample are of $A$-type, we need only to consider the amplitudes $A^{ \pm}$and $B^{ \pm}$. The normalization can be chosen by setting $A^{+}=1$ and $B^{-}=1$. For an incoming wave $\mathrm{e}^{\mathrm{i} K z} u(z)=A^{+} \mathrm{e}^{\mathrm{i} k_{A} z}+B^{+} \mathrm{e}^{-\mathrm{i} k_{A} z}$, the reflected and transmitted waves become $\bar{r} \mathrm{e}^{-\mathrm{i} K z} u(z)=\bar{r}\left(A^{-} \mathrm{e}^{\mathrm{i} k_{A} z}+B^{-} \mathrm{e}^{-\mathrm{i} k_{A} z}\right)$ and $\bar{t} \mathrm{e}^{\mathrm{i} K z} u(z)=\bar{t}\left(A^{+} \mathrm{e}^{\mathrm{i} k_{A} z}+B^{+} \mathrm{e}^{-\mathrm{i} k_{A} z}\right)$, respectively. Therefore, by setting $A_{0}=A^{+}+\bar{r} A^{-}, B_{0}=B^{+}+\bar{r} B^{-}, A_{2 L+2}=\bar{t} A^{+}$and $B_{2 L+2}=\bar{t} B^{+}$in (6), we find

$\bar{t}=\frac{\left(\mathcal{T}_{11} A^{+}+\mathcal{T}_{12} B^{+}\right)\left(\mathcal{T}_{21} A^{-}+\mathcal{T}_{22} B^{-}\right)-\left(\mathcal{T}_{21} A^{+}+\mathcal{T}_{22} B^{+}\right)\left(\mathcal{T}_{11} A^{-}+\mathcal{T}_{12} B^{-}\right)}{\left(\mathcal{T}_{21} A^{-}+\mathcal{T}_{22} B^{-}\right) A^{+}-\left(\mathcal{T}_{11} A^{-}+\mathcal{T}_{12} B^{-}\right) B^{+}}$

$\bar{r}=\frac{\left(\mathcal{T}_{11} A^{+}+\mathcal{T}_{12} B^{+}\right) B^{+}-\left(\mathcal{T}_{21} A^{+}+\mathcal{T}_{22} B^{+}\right) A^{+}}{\left(\mathcal{T}_{21} A^{-}+\mathcal{T}_{22} B^{-}\right) A^{+}-\left(\mathcal{T}_{11} A^{-}+\mathcal{T}_{12} B^{-}\right) B^{+}}$.

For all nine sets of parameters listed in table 1 and $\sigma=0.1$, we recalculate $R\left(=\bar{r} \bar{r}^{*}\right)$ and $T\left(=\bar{t} \bar{t}^{*}\right)$ according to (13) and (14) for various values of $q$ ranging from 0.1 to 10 . The two-parameter scaling behaviour is indeed recovered. For instance, for the case of $q=0.5$, we plot in figure 1 the probability distribution $P_{\Lambda}$ of the renormalized reflection coefficient $(R-1) / 2 q$ at $\Lambda=0.3,0.6$ and 3.0 for all nine sets of parameters considered here. The values of $\epsilon^{\prime \prime}$ for the case of $q=0.5$ are given in the last column of table 1 . Different symbols in figure 1 represent different sets of parameters. From figure 1, it is easy to see that all data collapse into curves $\mathrm{A}, \mathrm{B}$ and $\mathrm{C}$ for $\Lambda=0.3,0.6$ and 3.0, respectively. The functional forms of these curves are the same as those obtained from homogeneously random media, albeit with larger fluctuations [7]. The same is true for the transmission coefficient [7]. Also in figure 1, we plot in a heavy solid curve the analytic result of equation (9). It should be mentioned that the values of localization length $\xi_{0}$ in these nine cases range from 610 to 4050 (table 1). All these results demonstrate that, with the MBC, random systems that are periodic on average do obey the same univeral scaling behaviours for high frequencies.

\section{Gain length}

Although the relation between gain length $l_{\mathrm{g}}$ and $\epsilon^{\prime \prime}$ can be obtained numerically, an analytic relation exists when $\epsilon^{\prime \prime} \ll \epsilon_{A}$ and $\epsilon_{B}$. The value of $l_{\mathrm{g}}$ can be obtained from the inverse of the imaginary part of the Bloch wavevector. If we replace $k_{A}$ and $k_{B}$ in (11) by 


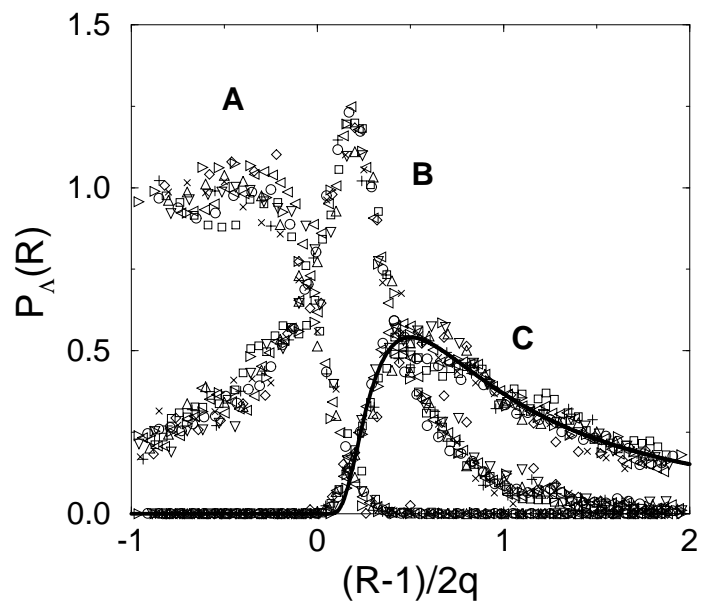

Figure 1. For the case of a matched boundary condition and $q=0.5$, the probability distribution $P_{\Lambda}$ of the reflection coefficient $R$ is plotted as a function of $(R-1) / 2 q$ for the renormalized length $\Lambda=0.3$ (curve A), 0.6 (curve B) and 3.0 (curve C). Different symbols represent data obtained from different sets of parameters listed in table 1 . The solid line in curve $\mathrm{C}$ is the analytic result when $\Lambda \rightarrow \infty$.

$k_{a}=(\omega / c) \sqrt{\epsilon_{A}-\mathrm{i} \epsilon^{\prime \prime}}$ and $k_{b}=(\omega / c) \sqrt{\epsilon_{B}-\mathrm{i} \epsilon^{\prime \prime}}$, respectively, and write $K=k+\mathrm{i} \kappa$, we have $l_{\mathrm{g}}=1 / \kappa$. In the case of small gain, we expand both sides of the complex secular equation to the first order in $\kappa$ and $\epsilon$. By comparing the real and imaginary parts separately, we find that

$$
\begin{aligned}
& k=\cos ^{-1}(\Omega) / d \\
& \kappa=\aleph \epsilon^{\prime \prime} / d \sqrt{1-\Omega^{2}}
\end{aligned}
$$

where

$$
\begin{aligned}
& \Omega=\cos \left(k_{A} a\right) \cos \left(k_{B} b\right)-\frac{k_{A}^{2}+k_{B}^{2}}{2 k_{A} k_{B}} \sin \left(k_{A} a\right) \sin \left(k_{B} b\right) \\
& \aleph=\frac{1}{2 \Xi}\left[\sqrt{\theta_{+}^{2}+\Theta^{2}} \sin \left(\alpha_{+}-\beta_{+}\right)+\sqrt{\theta_{-}^{2}+\Theta^{2}} \sin \left(\alpha_{-}-\beta_{-}\right)\right] \\
& \alpha_{ \pm}=k_{A} a \pm k_{B} b \\
& \beta_{ \pm}=\tan ^{-1}\left[\frac{\left(k_{A}^{2}-k_{B}^{2}\right)\left(\epsilon_{B}-\epsilon_{A}\right)}{\left(k_{B} \pm k_{A}\right)^{2}\left(\epsilon_{A} k_{B} b \pm \epsilon_{B} k_{A} a\right)}\right] \\
& \Theta=\left(k_{A}^{2}-k_{B}^{2}\right)\left(\epsilon_{B}-\epsilon_{A}\right) \\
& \theta_{ \pm}=\left(k_{A} \pm k_{B}\right)^{2}\left(\epsilon_{A} k_{B} b \pm \epsilon_{B} k_{A} a\right) \\
& \Xi=4 k_{A} k_{B} \epsilon_{A} \epsilon_{B} .
\end{aligned}
$$

The values of $l_{\mathrm{g}}$ obtained from (16) agree well with those obtained numerically for all the cases we study here. In the small- $k$ limit, it can be shown that equation (16) reduces to the homogeneous medium result, i.e. $\kappa \approx k \epsilon^{\prime \prime} / 2 \epsilon_{0}$ with $\epsilon_{0}=\left(a \epsilon_{A}+b \epsilon_{B}\right) / d$. Equation (16) also describes the attenuation length in the case of dissipation $\left(\epsilon^{\prime \prime}<0\right)$. 


\section{Saturation length}

Now we discuss the dependence of the boundary condition on the saturation length. It can easily be seen that that the numerically obtained distribution of $P_{3.0}$ shown in figure 1 (curve C) coincides with the analytic $P_{\infty}$. This indicates that the stationary distribution has already been reached when $\Lambda=3.0$. The value of $\Lambda$ where the distribution saturates to the stationary form of equation (9) can be determined quantitatively from the mean square deviation $\delta_{P}(\Lambda)$ from the analytic $P_{\infty}$ [7]. This function decreases linearly with $\Lambda$ before it saturates to a constant value, which decreases with the number of configurations. If we extrapolate the linear portion of the curve to the $\Lambda$-axis, the intercept can be considered as the saturation length $\Lambda_{\mathrm{s}}$. For the range of q considered here $(0.1<q<10)$, the saturation length is found to follow $\Lambda_{\mathrm{s}} \cong 0.7 q^{-1 / 2}$. This result agrees with that of homogenously random systems, being smaller only by a factor of 0.7 due to the difference in defining $\Lambda_{\mathrm{s}}$ [7]. For the case of MMBC, since we do not have a universal distribution $P_{\infty}$ to use, we simply replace it by $P_{3.0}$. The function $\delta_{P}(\Lambda)$ shows similar behaviour to the case of MBC. The saturation length obtained agrees well with that obtained from MBC. This explains why the method of measuring the localization length by using resonant tunnelling spectra can be applied to the experimental data [11].

\section{Discussions}

It should be mentioned that we have studied also the behaviour of localization length in the presence of gain. As in the case of homogeneously random systems, the localization length is found to be reduced and to follow a simple relation $1 / \xi=1 / \xi_{0}+1 / l_{\mathrm{g}}$ [7]. Since both $\xi_{0}$ and $l_{\mathrm{g}}$ are intrinsic quantities of the sample, the localization length in the presence of gain $\xi$ is also independent of the boundary condition. Although we have discussed only the case of amplification, similar results are expected for the case of absorption. It has been shown that for homogeneously random systems there exists a universal distribution for the reflection coefficient. Moreover, the localization length in the presence of absorption follows the same relation obtained for the case of amplification, except replacing the gain length by the attenuation length $[7,9,14]$. It should be mentioned that, physically, the reduction of the localization length in a gain medium is due to the enhancement of the coherent backscattering effect. In contrast, a similar reduction in an absorbing medium arises from the attenuation of waves in the forward direction. Mathematically, it has been shown recently that by using a dual symmetry between absorption and amplification the localization length in an amplifying medium is the same as that in an absorbing medium [8]. Since in real systems, absorption always exists when frequency is sufficiently high [15], a distribution of the reflection coefficient obtained experimentally by using a MMBC should always be non-universal. The 1D universal scaling behaviour can be recovered only by using a MBC.

Although all the results presented here are calculated for the case of small randomness ( $\sigma=0.1$ ), we have also studied the case when randomness is large. In general, we found that the scaling holds as long as the strength of scattering is such that the localization length in the absence of gain, $\xi_{0}$, is larger than 100 . For instance, for the first set of parameters in table $1\left(\epsilon_{A} / \epsilon_{B}=9 / 1\right.$ and $\left.\omega=0.35\right)$, the values of $\xi_{0}$ are about $400,130,80$ and 50 when $\sigma=0.3,0.4,0.5$ and 0.6 , respectively. For the cases $\sigma=0.3$ and 0.4 , the distribution functions, $P_{\Lambda}$, lie within the fluctuations of the curves $\mathrm{A}, \mathrm{B}$ and $\mathrm{C}$ shown in figure 1 . Deviations become obvious when $\sigma=0.6$. Similar results have been found in [7] for the case of homogeneously random systems. 
Finally, we would like to point out that the MBC applies only to frequencies in the bands. Therefore, it is not possible to have a universal scaling behaviour for frequencies inside the gaps, where the waves are evanascent in nature.

\section{Acknowledgments}

K-CC is grateful for the support by Sir Edward Youde Memorial Fellowship 95/96. The work was also supported in part by the Hong Kong Research Grant Council (RGC) under contract HKUST 617/95p.

\section{References}

[1] See, for example:

Sheng P (ed) 1990 Scattering and Localization of Classical Waves in Random Media (Singapore: World Scientific)

[2] See, for example:

Kempe M, Berger G A and Genack A Z 1996 Handbook of Optical Properties, vol II ed R E Hummel and P Wismann (Boca Raton, FL: Chemical Rubber Company)

[3] Zyuzin A Yu 1994 Europhys. Lett. 26517

[4] Wiersma D S, van Albada M P and Lagendijk Ad 1995 Phys. Rev. Lett. 751739

[5] Feng S C and Zhang Z Q 1996 Phys. Rev. B 543240

[6] Deng W, Wiersma D S and Zhang Z Q 1997 Phys. Rev. B 56178

[7] Zhang Z Q 1995 Phys. Rev. B 527960

[8] Paasschens J C J, Misirpashaev T Sh and Beenakker C W Phys. Rev. B 5411887 (1996).

[9] Pradhan P and Kumar N 1994 Phys. Rev. B 509644

[10] Deng W and Zhang Z Q 1997 Phys. Rev. B 555337

[11] Zhang Z Q, Chiu K C and Zhang D Z 1996 Phys. Rev. B 5411891

[12] Beenakker C W, Paasschens J C and Brouwer P W 1996 Phys. Rev. Lett. 761368

[13] See, for example:

Soukoulis C M 1996 Photonic Band Gap Materials (Dordrecht: Kluwer)

[14] McGurn A R, Christensen K T, Mueller F M and Maradudin A A 1993 Phys. Rev. B 4713120

[15] Zhang D Z, Hu W, Zhang Y L, Li Z L, Cheng B Y and Yang G Z 1994 Phys. Rev. B 509810

[16] See, for example:

Liboff R L 1992 Introductory Quantum Mechanics (Reading, MA: Addison-Wesley) 